\section{THE MIGRATORY APPENDIX}

\author{
J. E. ALLABEN, M.D. \\ Attending Surgeon, St. Anthony Hospital \\ ROCKFORD, ILL.
}

There are certain types of tumors in the abdomen, usually cystic in character, that become attached by adhesions at a point distant from their origin and finally become detached entirely from their original locality, their nutrition being maintained through blood vessels in the new adhesions. These tumors are known as migratory tumors. The peculiarity of these growths suggested to my mind the caption of this article. It is not difficult to understand that the appendix might be subjected to a similar condition, and, being separated from the cecum, might maintain an entirely separate life through new blood vessels formed in abundant adhesions. A condition of this kind is well illustrated by the following case:

\section{REPORT OF CASE}

Miss F. M., aged 23, had suffered from a gonorrheal infection for which I had treated her for nine months. In August, 1917, she had recovered'sufficiently so that treatments were discontinued. She reappeared in August, 1918, complaining of pain in the right iliac region. This pain became so acute that she was confined to bed. There was some nausea, elevation of temperature and increase of pulse rate. The patient complained of tenderness in the right iliac region on pressure. There was much difficulty in getting a bowel movement. On account of the previous history of tubal disease it was a question whether the trouble was pyosalpinx or appendicitis. I decided to make an exploratory examination, which I did.

I operated on her, Sept. 10, 1918, at Rockford City Hospital. The incision was made through the right rectus muscle, so that it might be enlarged in either direction to make free access to the appendix or tube as might be necessary. On opening the abdomen $I$ found a considerable mass of omentum adherent in the right iliac fossa. This I ligated en masse and removed it entirely. The intestines in this region were kinked and firmly adherent to each other, thus accounting for the partial intestinal obstruction. The intestines were covered with a yellow plastic exudate. Sterilized gauze was used in separating the adhesions. The search was made at the cecum for the appendix, but I was unable to locate it. The right fallopian tube was distorted and adherent. Pressure on the tube forced a few drops of pus out of the fimbriated extremely. The tube was ligated and removed. At this stage of the operation the distal end of the appendix was observed protruding about half an inch through a mass of adhesion in the right iliac fossa. The appendix was dissected out from these adhesions as thoroughly as possible. The toilet of the peritoneum was finished by pouring about 4 ounces of ether into the pelvis and right iliac fossa, so that any infection of this locality through the tube might be counteracted and the peritoneum closed in layers.

No doubt a continual leakage of pus from the right tube produced the intestinal adhesions, and the appendix, which had become entirely separated from the cecum, was kept alive through new blood vessels formed in the many adhesions about this organ.

The patient made a good recovery without complications, save a slight infection in one of the skin stitches.

\section{COMMENT}

It is difficult to tell whether the appendix or the tube was primarily the offending organ in this case. It is interesting to speculate as to what pathologic condition would have followed if such an appendix had been left entirely to nature. It is my own opinion that the appendix and the adhesions would ultimately have been absorbed without causing any trouble, but it is plainly to be seen that a tube with a patulous extremity periodically leaking pus into the peritoneum might have caused serious complications. This condition of the appendix may account for some of the cases reported in which no appendix was found.

\section{BRONCHOPNEUMONIA DUE TO HEMO- LYTIC STREPTOCOCCI FOLLOWING INFLUENZA *}

\author{
ERNEST W. GOODPASTURE, M.D. (Boston) \\ Lieutenant (Junior Grade), M. C., U. S. N. R. F. \\ CHELSEA, MASS.
}

The bacteriology and pathology of bronchopneumonia during the last two months (December and January) have become quite different, at the Chelsea Naval Hospital, from that found during the preceding three months, including the most severe period of the first local outbreak of influenza. In the earlier period, cultures of Pfeiffer's bacillus were obtained from the lungs in 86 per cent. of cases postmortem, the organism often being found in pure culture, pneumococci in 50 per cent., and hemolytic streptococci in 13 per cent.; while during the last two months, cultures of hemolytic streptococci have been obtained from the lungs in 100 per cent. of those examined at necropsy, and in the majority of instances pure cultures were obtained. Pfeiffer's bacillus was demonstrated in only two cases of a total of sixteen, and these two were examined the first week of December.

This marked variation in the type of invading organism in bronchopneumonia is of particular interest since it represents the experience of a single station during a relatively short period of time; and we are now familiar with wide differences in bacteriologic observations from various sections of the country since the epidemic of influenza began.

From the twenty-six cases studied during the height of the epidemic in the early fall, we became more or less familiar with a type of pneumonia which was fairly typical of that series, and were able to recognize many modifications obviously due to secondary invasion by pathogenic organisms. An acute bronchiolitis characterized by the presence within the bronchioles of a dry exudate consisting of a few leukocytes, poly:morphonuclear and mononuclear, mucus and fibrin, usually with partial or complete necrosis of the mucous membrane, and the presence of a characteristic irregular hyaline membrane on the infundibular and alveolar walls, were regarded as typical early lesions. Bronchopneumonia following this was fulminating in type and consisted in a widespread toxic injury to alveolar tissues, so that they became filled with fluid exudate, fibrin, and hemorrhage with extensive injury to alveolar walls and capillaries. From a study of these cases we stated that "the pulmonary injury and reaction being so acute and often widespread, and the fact that in certain very early cases bacteria of any kind are so scarce or not found at all, make us feel, notwithstanding the demonstration of influenza bacilli in pure culture in the lungs in all but one instance, that at this stage organisms are as yet comparatively few within alveoli, and their primary injury is due to a very potent toxic substance elaborated in and disseminated through larger air passages." Following

* From the U. S. Naval Hospital. 